\title{
Récentes Applications des Techniques Electrodiffusionnelles en Ecoulement Diphasique
}

par Bachir Benabes, Gérard Cognet, Géraldine Pascal

du Laboratoire des Ecoulements Géophysiques et Industriels (LEGI), CNRS de Grenoble

Serguei Martemianov

du Frumkin Institute of Electrochemistry,

Russian Academy of Sciences de Moscou

Vaclav Sobolik

de l'Institute of Chemical Process Fundamentals

Academy of Sciences de Pragues

I TRANSFERT A UNE INTERFACE LIQUIDE-LIQUIDE COMPARÉ A UNE INTERFACE LIQUIDE-SOLIDE

Les mesures du cisaillement et du coefficient de transfert de masse à l'interface solide-liquide selon la technique électrodiffusionnelle sont maintenant bien maitrisées [1]. Il a semblé utile d'étendre leurs applications à l'étude de l'hydrodynamique et du transfert de masse au travers d'une interface liquide-liquide qui présente un grand intérêt dans de nombreux procédés polyphasiques [2]. 
- La faisabilité de l'expérience a été vérifiée dans une cuve agitée $(\varnothing 100 \mathrm{~mm})$ dont le fond portait l'électrode de Gallium dans une cavité de $5 \mathrm{~mm}$ de diamètre à $25 \mathrm{~mm}$ de laxe de la cuve. La vitesse de rotation de l'agitateur à 4 pales pouvait varier de 0 à $7 \mathrm{tr} / \mathrm{s}$. (fig. $l a$ et $I b$ ). La solution électrochimique comportait une répartition équimolaire de Ferri et Ferrocyanure de Potassium $\left(10 \mathrm{moles} / \mathrm{m}^{3}\right)$ et de la Soude comme électrolyte support (100 moles $\left./ \mathrm{m}^{3}\right)$.

- Les courbes de polarisation courant-tension ont été tracées, pour différentes vitesses de rotation $\omega$ et différentes températures $T$, correspondant à l'état solide et à l'état liquide de l'électrode de Gallium.

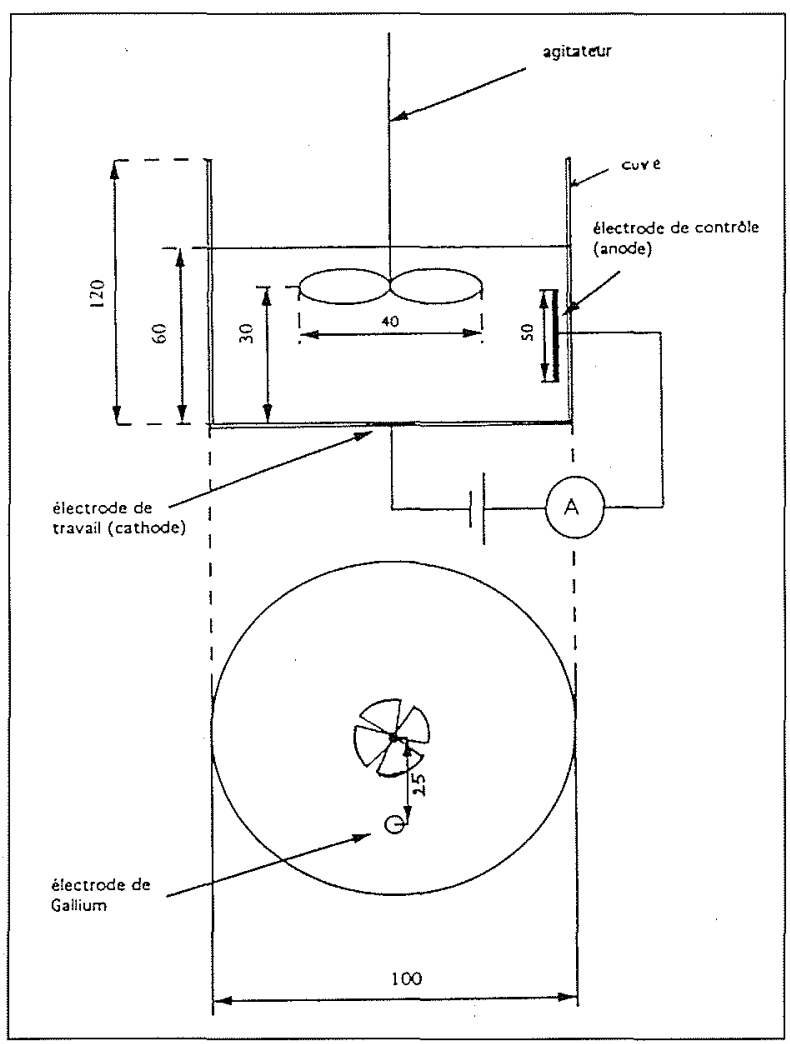

1a. Schéma expérimental.

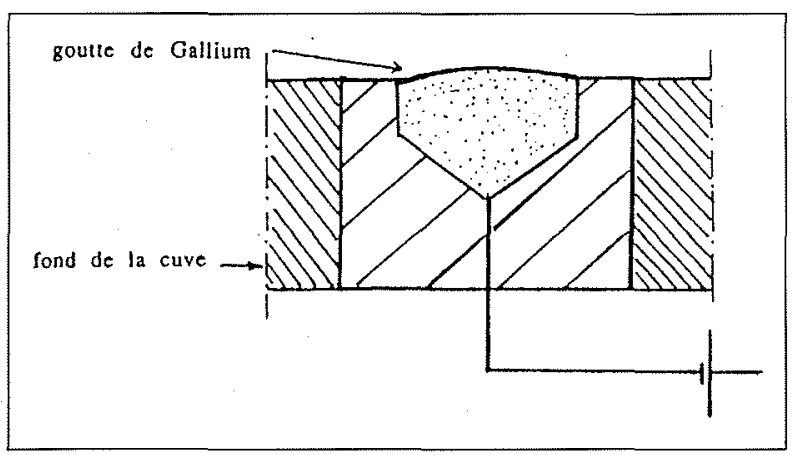

1b. Détail de l'électrode de Gallium.

Pour une température donnée la dépendance du courant limite $I$ avec la vitesse d'agitation $\omega$ (fig. 2 ) est représentée par une loi en puissance du type:

$$
I=\omega \cdot \omega^{N}
$$

On obtient $N=0,56$ que le Gallium soit liquide ou solide.

Le coeflicient $\alpha$ dépend de la température; il augmente avec $T$ jusqu’à la température de fusion, pour laquelle il décrô̂t brutalement de près de $20 \%$ avant d'augmenter à nouveau (fig. 3). On identifie ainsi précisément le changement de phase du Gallium. Il est probable que la diminution du transfert est liée aux mouvements de recirculation internes à la goutte de Gallium liquide sous la contrainte de l'écoulement de la solution agitée dans la cuve. Le résultat du passage à la phase liquide aurait pour effet de réduire sensiblement le cisaillement $\gamma$ à la paroi de l'électrode dont on sait qu'il est déterminant pour le transfert de masse. Etant donné que $I$ est pratiquement proportionnel à $\gamma^{1 / 3}$, en restant à une analyse d'ordre de grandeur, il vient d'après (1) :

$$
\gamma \sim \omega^{1,68}
$$

ce qui est compatible avec un écoulement agité moyennement turbulent dans la cuve.

Une diminution de $20 \%$ du transfert représenterait ainsi une réduction de plus de $60 \%$ du cisaillement à l'interface de l'électrode entre l'état solide et l'état liquide.

Ce résultat semble cohérent avec le fait que la viscosité cinématique du Gallium liquide est d'environ $0,310^{-6} \mathrm{~m}^{2} / \mathrm{s}$ dans le domaine de température concerné c'est-à-dire le tiers de celle de l'eau qui a donc un effet d'entrainement notable sur la recirculation de la goutte de métal liquide. Cette analyse mérite à l'évidence un développement plus quantitatif.

\section{Conclusion :}

Nous pensons ainsi avoir montré l'intérêt de l'application des techniques électrochimiques aux métaux liquides soit, comme on l'a considéré ici, pour représenter et qualifier les phénomènes de transport aux interfaces liquideliquide soit comme outil d'approche et de diagnostic du mouvement des métaux liquides.

Ces deux voies pourront être explorées dans l'avenir.

\section{SONDE PARIÉTALE TRISEGMENTÉE POUR L'ÉTUDE DE L'ÉCOULEMENT DE COUETTE-TAYLOR D'UNE SUSPEN- SION AQUEUSE DE FIBRES}

Les interactions entre les particules et le fluide dans les écoulements de suspension peuvent donner lieu à des phénomènes d'instabilité et de promotion de la turbulence ou dans certains cas de réduction de frottement. S'agissant de particules fibreuses il y a possibilité de floculation par enchevêtrement des fibres entre elles dans le champ de cisaillement [3]. Le dispositif de Couette entre cylindres coaxiaux constitue, à ce titre, un écoulement modèle. Pour un fluide newtonien des instabilités tridimensionnelles (cellules de Taylor) apparaissent au-delà d'une vitesse critique du cylindre intérieur, lorsque le cylindre extérieur reste fixe.

La présence des fibres modifie le comportement de la suspension par rapport au fluide support et le critère d'ins- 


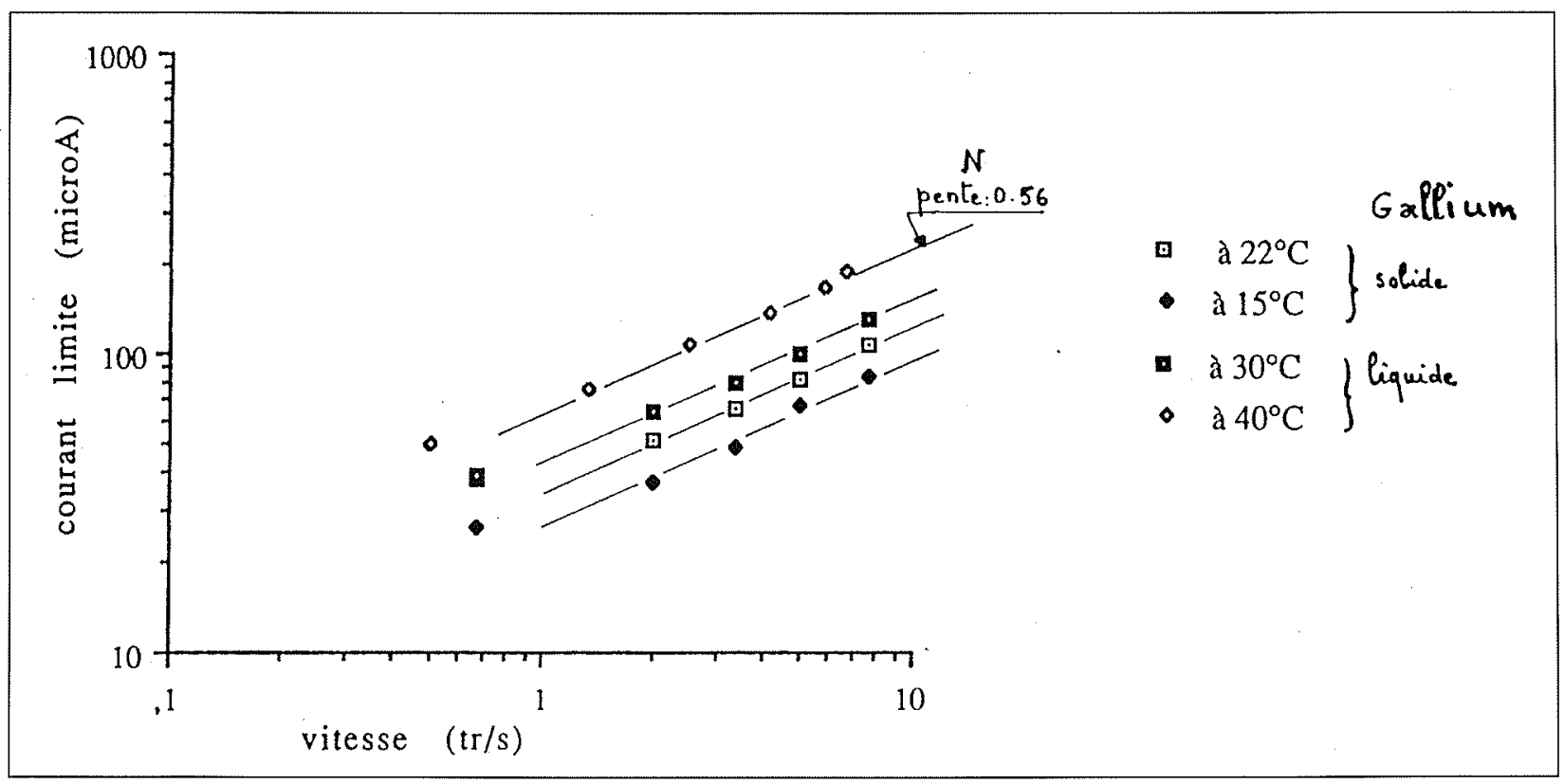

2. Courant limite fonction de la vitesse de rotation de l'agitateur à différentes températures.

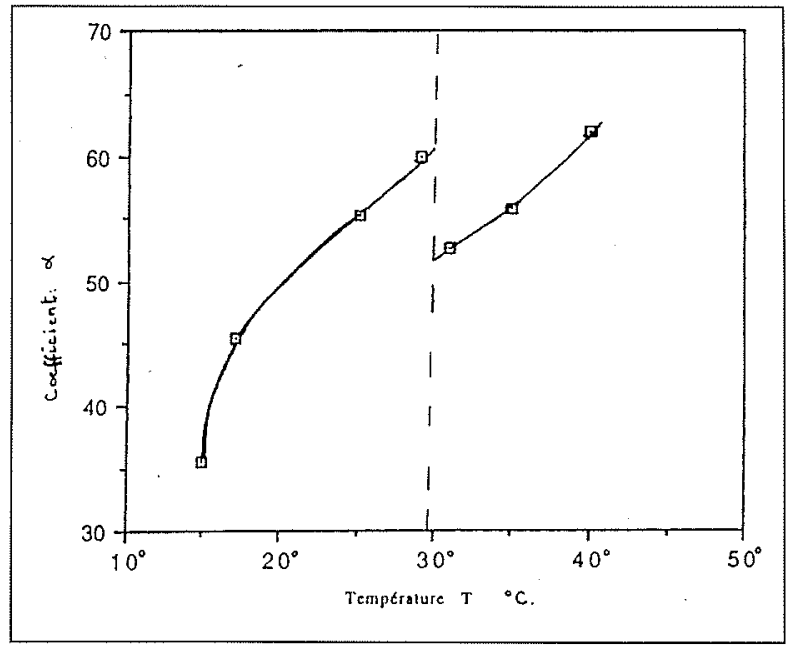

3. Coefficient $\alpha$ fonction de la température pour une vitesse d'agitation donnée $\omega=2 \mathrm{tr} / \mathrm{s}$.

tabilité correspondant ; on s'est intéressé à cet effet induit observé même à faible concentration.

L'étude a porté sur la détermination du nombre de Taylor critique et sur la croissance des cellules dans le cas d'une suspension de fibres. La méthode expérimentale s'est fondée sur la mesure des composantes azimuthale et axiale du cisaillement pariétal obtenues à l'aide d'une sonde électrochimique trisegmentée affleurant la surface du cylindre extérieur [4].

Les figures $4 a$ et $4 b$ montrent respectivement la sonde, constituée de 3 secteurs isolés de $120^{\circ}$ et son positionnement vis-à-vis d'une cellule de Taylor d'où il résulte une orientation us par rapport aux lignes de courant extrapolées à la paroi.
Un tel capteur présente une bonne sensibilité à la direction de l'écoulement; on le constate sur la figure 5 qui décrit les enregistrements du flux au niveau de chaque secteur de la sonde obtenus en opérant un tour complet de celle-ci autour de son axe; on peut ainsi déterminer précisément les composantes axiale $\gamma_{z}$ et azimuthale $\gamma_{\theta}$ du cisaillement du fluide à la paroi.

- Le dispositif expérimental comporte un ensemble de deux cylindres coaxiaux de rayons respectifs $\mathrm{R}_{1}=21,4 \mathrm{~mm}$ et $\mathrm{R}_{2}=30,6 \mathrm{~mm}$ et de hauteur $\mathrm{H}=140 \mathrm{~mm}$. Le cylindre intérieur est entraîné en rotation par un moteur à vitesse réglable entre 0,01 et $1 \mathrm{tr} / \mathrm{s}$.

La sonde trisegmentée située à mi-hauteur des cylindres, de diamètre $0,54 \mathrm{~mm}$, est entourée d'un tube inox de $3 \mathrm{~mm}$ qui sert d'électrode auxiliaire [4]. La solution électrolytique comprend un mélange équimolaire de Ferri et FerroCyanure de Potassium comme ions actifs et du iodure de Potassium comme électrolyte support lequel a l'avantage d'une forte densité ce qui permet d'ajuster sa concentration pour que la solution ait une masse volumique égale à celle des fibres de polyamide utilisées pour la suspension ( $\rho=1290 \mathrm{~kg} / \mathrm{m}^{3}$ - longueur $0,5 \mathrm{~mm}$ - diamètre $17 \mu \mathrm{m}$ ) avec $0,6 \%$ de concentration en volume; on évite ainsi le biais introduit par les phénomènes de sédimentation.

On peut provoquer un lent défilement en bloc de l'ensemble du système cellulaire devant la sonde par injection ou pompage de liquide à travers un orifice situé au fond de l'appareil et relié à une seringue motorisée ( $f$ ig. 6). On détermine ainsi l'évolution axiale du cisaillement en paroi à l'aide d'une seule sonde.

- La représentation des valeurs moyennes du cisaillement s'effectue dans le plan $\left(\gamma_{\theta}, \gamma_{z}\right)$ pour chaque état d'écoulement. En régime laminaire stable la composante $\gamma$, est nulle, le point représentatif se trouve sur l'axe des abscisses. L'apparition des cellules de Taylor se manifeste par l'existence d'une composante axiale du cisaillement conséquence du caractère tridimensionnel de la perturba- 


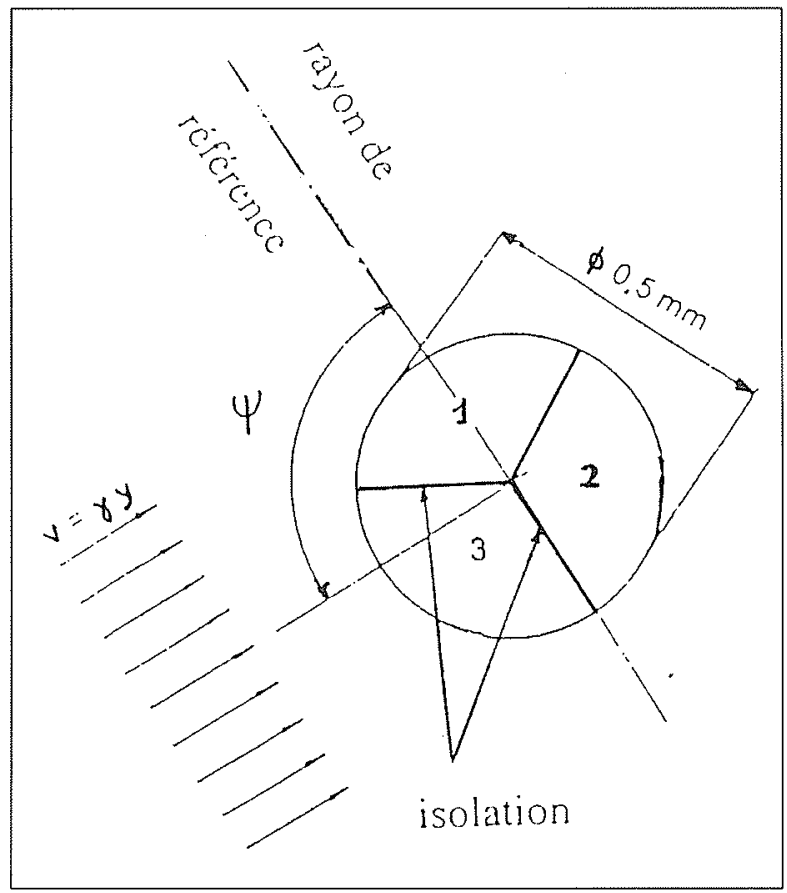

4a. Schéma de l'électrode trisegmentée.

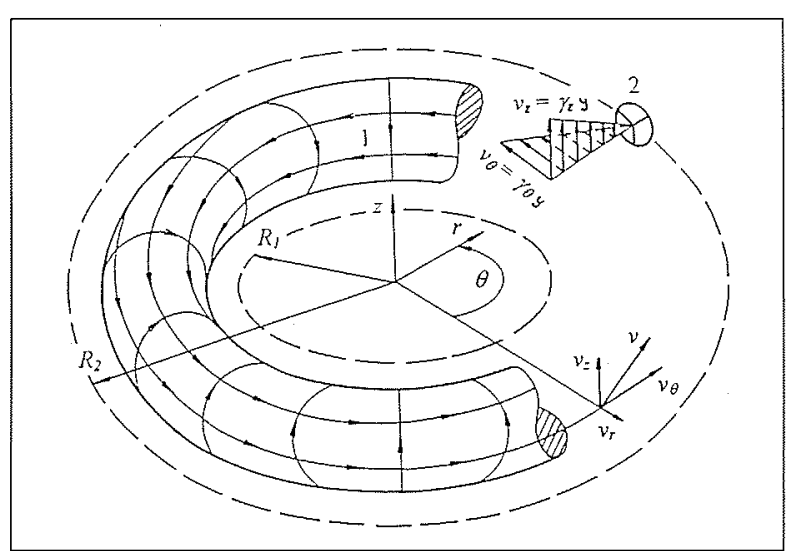

4b. Positionnement vis-à-vis d'une cellule de Taylor $y=R_{2}-r$.

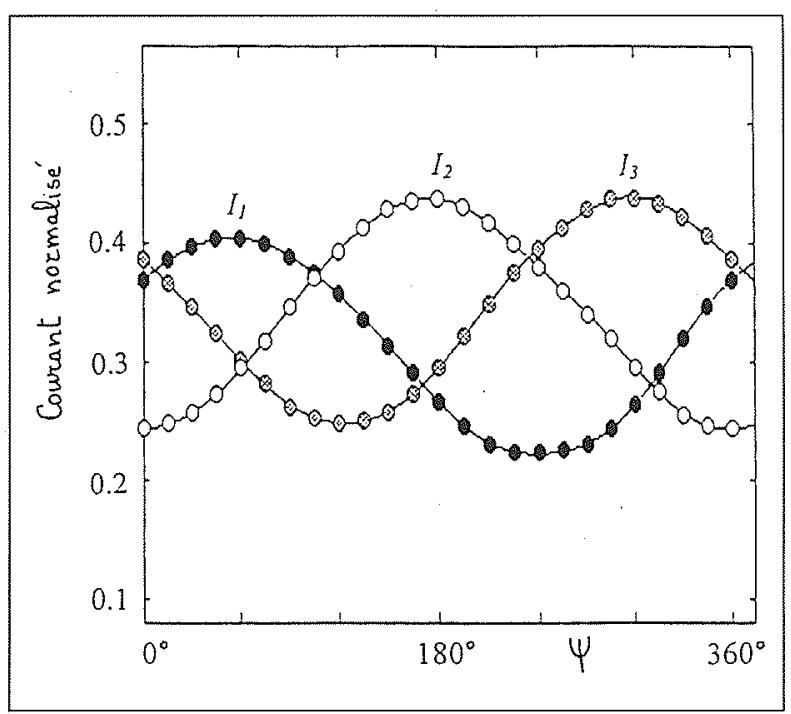

5. Courant recueilli sur chaque secteur d'électrode en fonction de l'angle d'orientation $\psi$.

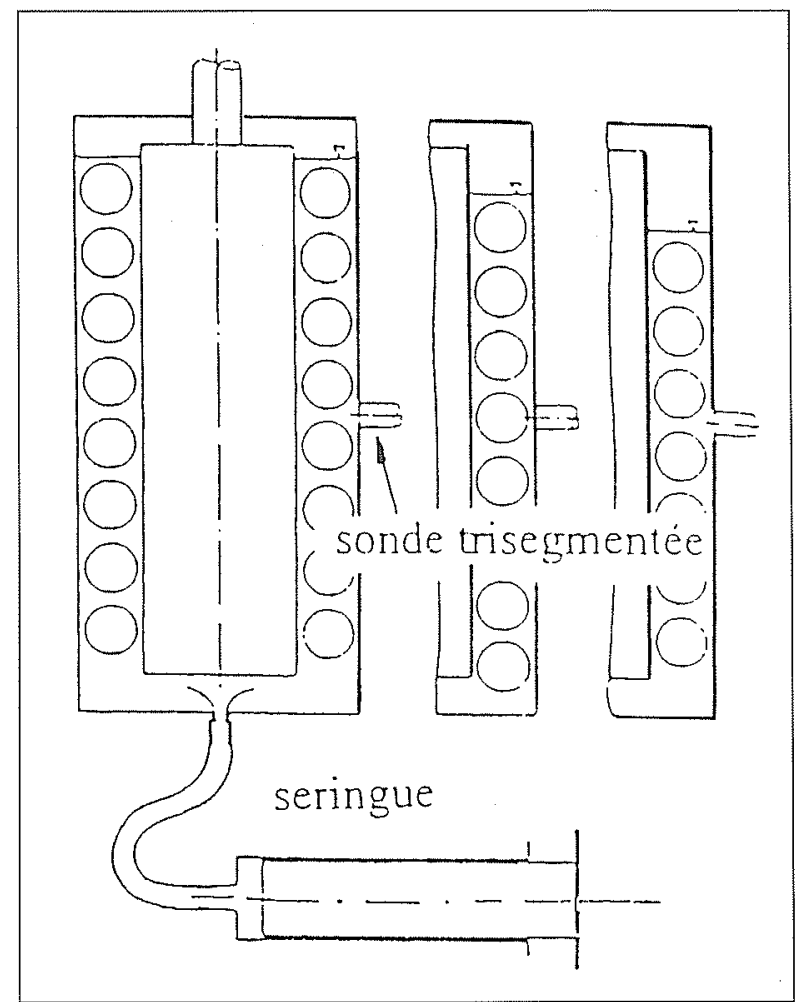

6. Schéma du dispositif permettant le défilement des structures cellulaires.

tion. La courbe fermée de la figure 7 illustre le défilement complet devant la sonde d'une paire de cellules pleinement développées dans des conditions quasi stationnaires. La forme en «coquille » qui est observée est caractéristique d'un large espace annulaire où se manifeste une forte non-linéarité spatiale de la perturbation par rapport au mouvement initial [5].

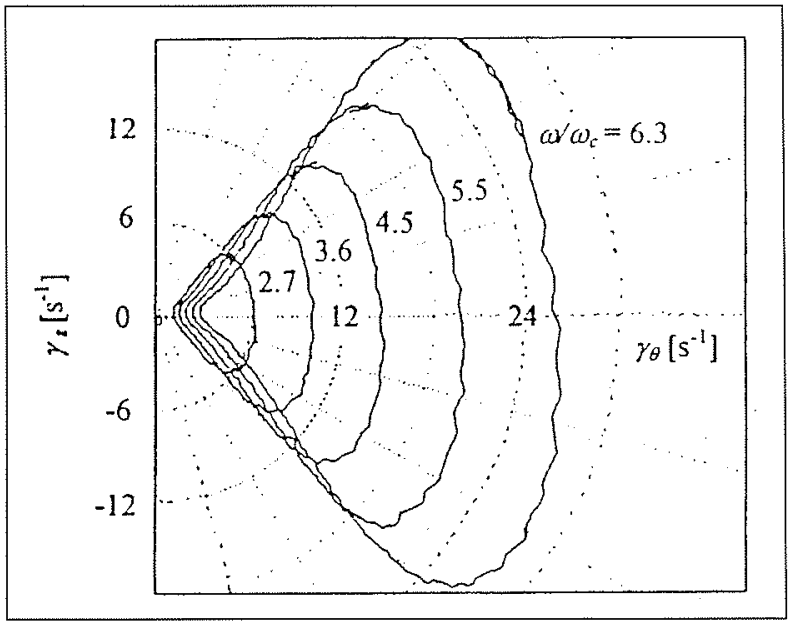

7. Représentation du cisaillement local par ses composantes axiale $\gamma_{-}$et azimuthale $\gamma_{\theta}$ pour différentes vitesses de rotation $\omega$. 
- La figure 8 présente l'évolution selon z des composantes du cisaillement dans l'hypothèse, toujours satisfaite, où le très laible débit axial, nécessaire au défilement devant la sonde de mesure, ne modifie pratiquement pas la géométrie ni la dynamique des structures cellulaires.

On constate que les courbes se distinguent sensiblement de la forme sinusö̈dale dès que la vitesse angulaire du cylindre 0 dépasse deux fois la valeur critique $\omega_{c}$. Dès lors il faut au minimum deux harmoniques pour représenter le mouvement secondaire. Ceci est particulièrement vrai pour la composante axiale de la perturbation [5]. On note également qu'il peut exister un très grand rapport entre la valeur maximale et la valeur minimale de la composante azimuthale du cisaillement correspondant respectivement aux zones « source» et «puit » de l'écoulement perturbé. Ce rapport peut atteindre 10 comme on l'observe pour $\omega / 0 .=5,5(f i g .8)$.

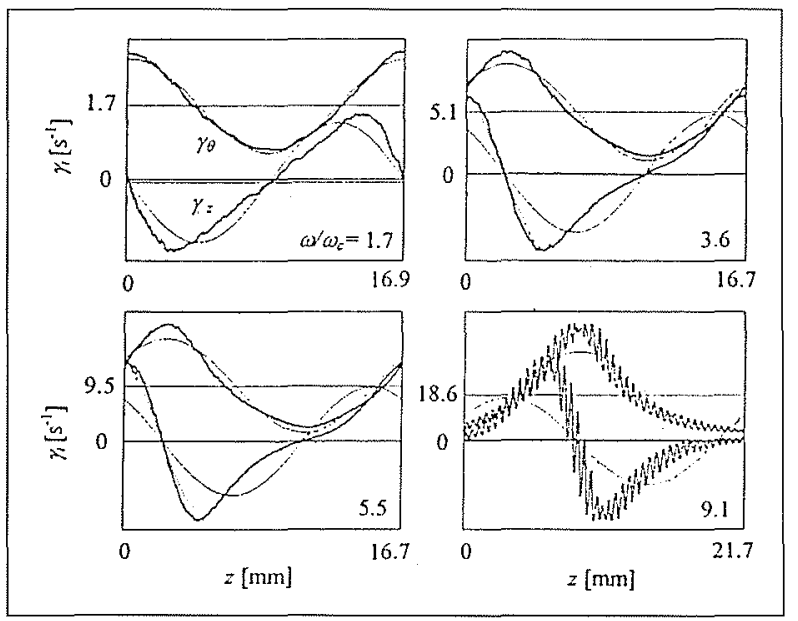

8. Distribution axiale de $\gamma_{0}$ et $\gamma_{\bar{z}}$ pour différentes vitesses de rotation $\omega$.

Enfin pour $\omega / \omega_{c} .=9,1$ le second mode d'instabilité relatif aux ondes azimuthales est clairement identifié par les fluctuations du signal autour de la courbe moyenne.

On a pu ainsi montrer [6] qu'avec ce type de fibres rigides de petites dimensions $(500 \mu \mathrm{m})$ et en faible concentration $(0,6 \%)$, l'effet résultant était un léger accroisse- ment de la stabilité par rapport à l'eau prise comme liquide support; la séquence des transitions vers l'écoulement de Taylor turbulent était par ailleurs plus facile à caractériser et d'une meilleure reproductibilité.

L'effet réducteur de frottement et de l'intensité de turbulence apparaît manifeste pour une telle suspension diluée.

\section{Conclusion :}

La mise en ouvre d'une sonde trisegmentée est un outil pour la description d'écoulements complexes au voisinage des parois. Tel est le cas des écoulements polyphasiques où l'interaction de la phase dispersée et de la phase continue a des conséquences essentielles sur les phénomènes et les propriétés de transferts pariétaux.

\section{Remerciements}

Les auteurs remercient le MRES et la Région RhôneAlpes qui ont permis d'effectuer ce travail au LEGI par lobtention d un poste PAST pour S. Martemianov et un support financier TEMPRA pour V. Sobolik.

Le theme de cette recherche s'inscrit dans le programme du GdR CNRS : GREDIC qui lui a accorde son soutien.

\section{Références}

[1] Hankattry T.J., Campbell J.A. (1983). - Fluid Mechanics Measurements. Ed. R.J. Goldstein, Hemisphere, p. 359.

2) Cognet G., Martemanov S. Pascal. G., Sobolik V. (1995). - C.R. Acad. Sc. Paris 1. 320, Série ll b, p. 505.

[3] Skalllami S., COGNET G., QUEmada D. (1985). -J.M.T.A $N^{\circ}$ spécial, p. 253.

[4] Destouls C., Gil O., SObOLIK V. (1990). - Int. J. Heat Mas. Tiansfer: 33, p. 1363.

15] Benabes B., Sobolik V., Cognet G. - Sélection des hamoniques et des amplindes dans un écoulement de ConetteTaylor 12 ${ }^{\circ}$ Congrè Français de Mécanique, Strasbourg, Sept 1995, Vol. 3, p. 245.

[6] BeNABES B. (1995). - Contribution à l'étude de la stabilité d'une suspension de fibres en écoulentent de Conetre-Taylor; Doctorat INP Grenoble. 


\title{
LA LOIRE
}

\section{Le fleuve et la vie \\ Tours, 13 et 14 juin 1996}

\section{Colloque de la SOCIÉTÉ HYDROTECHNIQUE DE FRANCE}

\begin{abstract}
A l'instigation du Ministère de l'Environnement (Direction de l'Eau) et avec le concours actif de l'Agence de l'eau LoireBretagne, la SOCIÉTÉ HYDROTECHNIQUE DE FRANCE, qui organise cette importante manifestation, a pour objectif d'apporter un éclairage scientifique aux consensus qui se font jour actuellement sur ce qu'il convient de faire ou ne pas faire tout au long de cette vallée prestigieuse, joyau des paysages de France. Avec la participation d'éminentes personnalités, seront examinés et débattus les aspects historiques, géographiques et géologiques, les questions que soulèvent la protection contre les inondations et le soutien des étiages et plus généralement tout ce qui intéresse le milieu et l'environnement : problèmes spécifiques à l'estuaire, urbanisation, extraction des matériaux, conservation des zones humides, restauration des populations de poissons migrateurs, qualité des eaux.
\end{abstract}

Ce colloque se propose d'offrir, aux responsables des administrations, des collectivités et des entreprises, aux animateurs des associations et à toutes les personnes intéressées par l'avenir de ce grand fleuve et de ses affluents un forum où ils pourront compléter leurs connaissances, débattre d'idées nouvelles et échanger leurs points de vue au cours de 3 sessions et d'une table ronde.

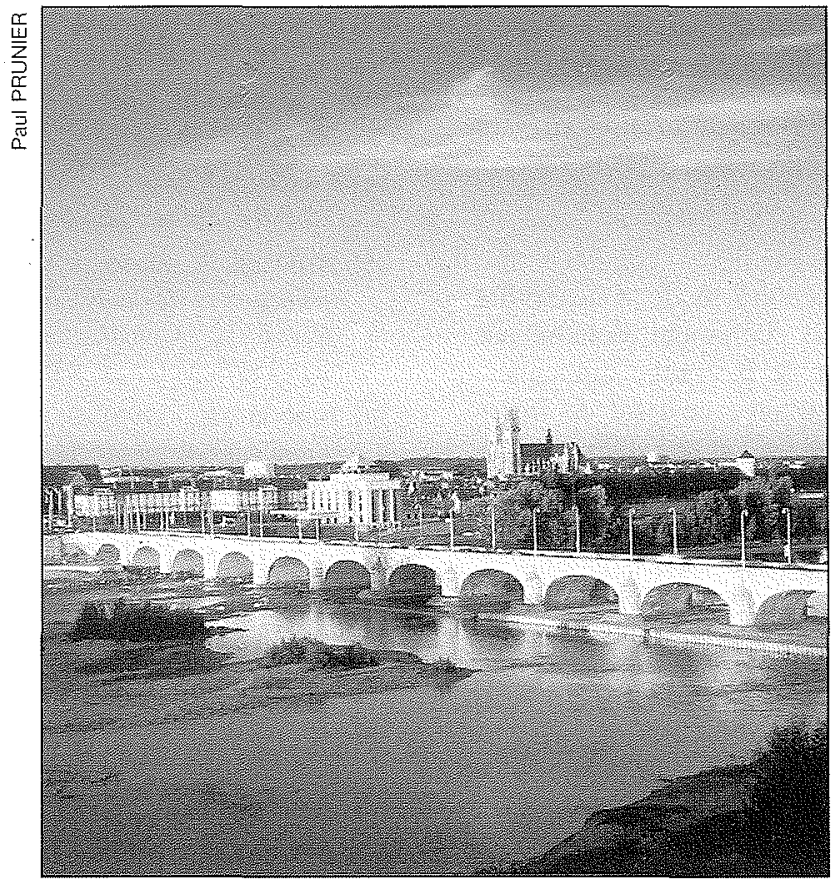

La Loire à Tours

Programne, renseignements et inscriptions

à la S.H.F.,

199, rue de Grenelle, 75007 Paris.

Tél. : (1) 47.05.13.37 - Fax : (1) 45.56.97.46. 